\title{
Nonlinear dynamics of Rossby waves in a western boundary current
}

\author{
L. J. Campbell \\ School of Mathematics and Statistics, Carleton University, \\ Ottawa, Canada
}

\begin{abstract}
This paper examines the nonlinear dynamics of a Rossby wave propagating longitudinally in a north-south shear flow. The flow configuration is an idealized model for a western boundary current in an ocean basin. It is assumed that there is a critical layer in the flow, where the shear flow speed is the same as the wave phase speed. The nonlinear critical-layer evolution of the wave depends on the direction of propagation of the wave. Numerical simulations show that an eastwardpropagating wave incident on the critical layer from the west is absorbed by the mean flow at early times. This is the same situation that is known to occur for small-amplitude waves, according to the linear theory. At later times, however, nonlinear waves may be reflected from the critical layer. In contrast, a westwardpropagating wave incident on the critical layer from the east passes through largely unaffected. An approximate analytic solution of the linearized equations is also presented to give further insight into the evolution of the critical layer.

Keywords: critical layer, Rossby waves, nonlinear wave interactions, western boundary current, shear flow, numerical simulations.
\end{abstract}

\section{Introduction}

An idealized model for the dynamics of Rossby waves in a western boundary current in an ocean basin consists of a latitudinally-periodic Rossby wave propagating horizontally in the zonal direction in a north-south shear flow $[6,7,8]$. This paper examines the nonlinear interactions between the waves and the current in the vicinity of a critical layer. A critical layer is a region surrounding a longitude at which the shear flow velocity is equal to the phase speed of the wave. When the governing linearized inviscid equations are solved numerically or analytically it is seen that 
the behaviour of a small-amplitude wave incident on the critical layer depends on the direction of propagation of the wave. An eastward-propagating wave incident on the critical layer from the west is absorbed, i.e. its momentum is transferred to the mean flow, but a westward-propagating wave forced to the east of the critical layer passes through without attenuation [3, 7].

However, the fate of large-amplitude waves which are governed by nonlinear theory remains unclear. The question is whether a nonlinear wave incident on a critical layer in a north-south flow would be absorbed or transmitted as in the linear theory. Another possibility is that the wave would be reflected at the critical layer. This is the situation that occurs in the related problem in which a Rossby wave, periodic in the zonal direction, propagates southwards towards a critical latitude in a zonal shear flow. In that configuration, it is well-known that, according to the linear theory, waves incident on the critical layer are completely absorbed $[5,10]$. Large-amplitude (nonlinear) waves are absorbed at the critical layer at early times; however, the nonlinear effects eventually become important and the critical layer then becomes a reflector of the incident waves $[1,9,11]$.

In the present study, the governing nonlinear equations for a longitudinallypropagating Rossby wave in a north-south shear flow are solved numerically in order to determine whether the wave is absorbed, reflected or transmitted. Both eastward- and westward-propagating waves are considered. It is found that an eastward-propagating wave incident on the critical layer from the west is absorbed by the mean flow at early times, but at later times there is wave reflection. On the other hand, a westward-propagating wave incident on the critical layer from the east passes through unaffected; this is the same situation that occurs in the linear theory. An approximate analytic solution of the linearized equations is also presented.

\section{Formulation}

The governing equation in this study is the barotropic vorticity equation. It is written in terms of non-dimensional variables as

$$
\nabla^{2} \Psi_{t}+\Psi_{x} \nabla^{2} \Psi_{y}-\Psi_{y} \nabla^{2} \Psi_{x}+\beta \Psi_{x}-R e^{-1} \nabla^{4} \Psi+B(x)=0,
$$

where $\Psi(x, y, t)$ is the total streamfunction and the subscripts denote partial differentiation with respect to time $t$ and the two space variables $x$ (longitude) and $y$ (latitude). The parameter Re is the Reynolds number and $\beta$ is the non-dimensional gradient of planetary vorticity. The term $B(x)$ represents a body force such as that due to topography and is included in order that the basic flow quantities, which are functions of $x$, can satisfy the governing equation. The Laplacian operator in (1) is non-dimensional with the $y$-derivative in the operator being multiplied by a factor $\delta=L_{x}^{2} / L_{y}^{2}$, where $L_{x}$ and $L_{y}$ are typical length scales in the zonal and meridional directions respectively. The parameter $\delta$ is the square of the aspect ratio.

The streamfunction is written as

$$
\Psi(x, y, t)=\bar{\psi}(x)+\varepsilon \psi(x, y, t) .
$$


where $\bar{\psi}$ is the streamfunction of the meridional basic flow and $\psi$ is the disturbance streamfunction. The basic flow is taken to be the initial $y$-independent flow. It is assumed that both $\bar{\psi}$ and $\psi$ are $O(1)$, so that the parameter $\varepsilon$ gives a measure of the magnitude of the perturbation relative to that of the basic flow. The basic velocity is related to the basic streamfunction by

$$
\bar{v}(x)=\bar{\psi}^{\prime}(x)
$$

where the prime denotes differentiation with respect to $x$. In the rest of this paper, the body force term in (1) is set to $B(x)=-\beta \bar{v}(x)$, in order that the basic streamfunction $\bar{\psi}(x)$ satisfies (1).

The assumption (2) leads to the nonlinear equation

$$
\left(\frac{\partial}{\partial t}+\bar{v} \frac{\partial}{\partial y}\right) \nabla^{2} \psi+\beta \psi_{x}-\bar{v}^{\prime \prime} \psi_{y}+\varepsilon\left(\psi_{x} \nabla^{2} \psi_{y}-\psi_{y} \nabla^{2} \psi_{x}\right)=0
$$

If it is assumed that $\varepsilon \ll 1$, one is justified in neglecting the nonlinear terms in this equation. This gives

$$
\left(\frac{\partial}{\partial t}+\bar{v} \frac{\partial}{\partial y}\right) \nabla^{2} \psi+\beta \psi_{x}-\bar{v}^{\prime \prime} \psi_{y}=0
$$

By writing the disturbance streamfunction in the neutral mode form

$$
\psi(x, y, t)=\operatorname{Re}\left\{\phi(x) e^{i l(y-c t)}\right\}
$$

where $l$ is the meridional wavenumber and $c$ is the phase speed, the following amplitude equation is derived:

$$
(\bar{v}-c)\left(\phi_{x x}-\delta l^{2} \phi\right)-\frac{i \beta}{l} \phi_{x}-\bar{v}^{\prime \prime} \phi=0 .
$$

This equation is singular at any point $x=x_{c}$ where $\bar{v}(x)=c$. This is the critical line and the region surrounding it is the critical layer. Using the method of Frobenius and expanding about the point $x_{c}$, it can be shown that two linearly independent power series solutions of this equation are

$$
\phi_{a}\left(x-x_{c}\right)=1+\frac{i l \bar{v}_{c}^{\prime \prime}}{\beta}\left(x-x_{c}\right)+\ldots
$$

and

$$
\phi_{b}\left(x-x_{c}\right)=\left(x-x_{c}\right)^{1+i \gamma}+\frac{\bar{v}_{c}^{\prime \prime}}{2 \bar{v}_{c}^{\prime}} \frac{(2-i \gamma(1+i \gamma))}{2+i \gamma}\left(x-x_{c}\right)^{2+i \gamma}+\ldots,
$$

where $\gamma=\beta / \delta l \bar{v}_{c}^{\prime}$ and the subscript $c$ denotes evaluation of $\bar{v}$ and its derivatives at $x_{c}$. The solution $\phi_{b}$ is singular at the point $x=x_{c}$. East and west of the singular 
point, $\phi_{b}$ can be written as

$$
\phi_{b}^{+}\left(x-x_{c}\right)=\left(x-x_{c}\right) e^{i \gamma \log \left|x-x_{c}\right|}+\ldots,
$$

and

$$
\phi_{b}^{-}\left(x-x_{c}\right)=\left(x-x_{c}\right) e^{-|\gamma| \theta} e^{i \gamma \log \left|x-x_{c}\right|}+\ldots,
$$

respectively, if, when $x<x_{c}$, the logarithm is defined to be

$$
\log \left(x-x_{c}\right)=\log \left|x-x_{c}\right|+i \theta \operatorname{sgn}\left(\bar{v}_{c}^{\prime}\right),
$$

with $\theta=-\pi$. Thus, the amplitude of the $\phi_{b}$ solution decreases by a factor of $e^{-|\gamma| \pi}$ as the wave crosses the critical line from west to east.

Using group velocity arguments, it can be shown [7] that the nonsingular solution $\phi_{a}$ corresponds to an westward-propagating wave. It has a long zonal wavelength and is non-divergent. The discontinuous solution $\phi_{b}$ corresponds to an eastward-propagating wave with short zonal wavelength and its momentum flux divergence is in general nonzero. Thus, the behaviour of the solution depends on whether the perturbation is forced to the west or east of the critical layer.

In the rest of this paper, the amplitude of the disturbance is assumed to be timedependent. In section 3, linear time-dependent solutions are presented and we shall see that these solutions satisfy properties analogous to those of the corresponding steady solutions (7) and (8). The results of some nonlinear numerical simulations are presented in section 4 .

\section{Analytic solution of the linear time-dependent equation}

Writing the disturbance streamfunction as

$$
\psi(x, y, t)=\operatorname{Re}\left\{\phi(x, t) e^{i l y}\right\}
$$

in the linear equation (4) gives the amplitude equation

$$
\left(\frac{\partial}{\partial t}+i l \bar{v}\right)\left(\phi_{x x}-\delta l^{2} \phi\right)+\beta \phi_{x}-i l \bar{v}^{\prime \prime} \phi=0 .
$$

The domain of definition of the solution is assumed to be semi-infinite in both time and space, i.e. $t \geq 0$ and either $-\infty<x \leq x_{1}$ or $x_{1} \leq x<\infty$. At the forced boundary $x=x_{1}$, the perturbation streamfunction is set to

$$
\psi\left(x_{1}, y, t\right)=\operatorname{Re}\left\{e^{i l(y-c t)}\right\},
$$

which means that

$$
\phi\left(x_{1}, t\right)=e^{-i l c t} .
$$

On defining the Laplace transform of $\phi(x, t)$ by

$$
\tilde{\phi}(x, s)=\int_{0}^{\infty} \phi(x, t) e^{-s t} d t
$$


and taking the transform of each term in (13), one obtains

$$
(s+i l \bar{v})\left(\tilde{\phi}_{x x}-\delta l^{2} \tilde{\phi}\right)+\beta \tilde{\phi}_{x}-i l \bar{v}^{\prime \prime} \tilde{\phi}=0
$$

with the boundary condition

$$
\tilde{\phi}\left(x_{1}, s\right)=\frac{1}{(s+i l c)} .
$$

The solution of (17) is a linear combination of the power series $\phi_{a}\left(x-x_{s}\right)$ and $\phi_{b}\left(x-x_{s}\right)$, where $x_{s}$ is the point where $\bar{v}(x)=-i s / l$, and $\phi_{a}$ and $\phi_{b}$ are defined in (7) and (8) respectively. For the special case where the aspect ratio $\delta$ is zero and the basic velocity is $\bar{v}(x)=x$, the functions $\phi_{a}$ and $\phi_{b}$ are simply equal to the leading order terms in each of their respective series, i.e. $\phi_{a}=1$ and $\phi_{b}=\left(x-x_{s}\right)^{1+i \gamma}$.

On applying the boundary condition and inverting the Laplace transform, $\phi$ is found to be

$$
\phi(x, t)=\frac{1}{2 \pi i} \int_{\alpha-i \infty}^{\alpha+i \infty} \frac{e^{s t}}{(s+i l c)} \frac{a(s) \phi_{a}\left(x-x_{s}\right)+b(s) \phi_{b}\left(x-x_{s}\right)}{a(s) \phi_{a}\left(x_{1}-x_{s}\right)+b(s) \phi_{b}\left(x_{1}-x_{s}\right)} d s,
$$

where the real constant $\alpha$ is chosen so that the contour of integration will lie to the right of all the singularities of the integrand. The functions $a(s)$ and $b(s)$ depend on the boundary conditions and determine the direction of propagation of the waves. For the case where $b(s)=0$, the only singularity of the integrand is the pole at $s=-i l c$, so the integral is evaluated by a simple residue calculation and the solution is found to be

$$
\phi(x, t)=\lim _{s \rightarrow-i l c}\left[e^{s t} \frac{\phi_{a}\left(x-x_{s}\right)}{\phi_{a}\left(x_{1}-x_{s}\right)}\right]=e^{-i l c t} \frac{\phi_{a}\left(x-x_{c}\right)}{\phi_{a}\left(x_{1}-x_{c}\right)} .
$$

Thus, the solution in this case is simply the steady westward-propagating solution (7) multiplied by a periodic function of $t$. The amplitude of the wave is unaffected by an encounter with a critical layer, there is no $-\pi$ phase change, and the solution does not contribute to the nonlinear dynamics of the critical layer.

If $b(s) \neq 0$, then the integral is equal to the sum of the contributions from three singularities: the pole at $s=-i l c$, and the branch points at $s=-i l x$ and $s=-i l x_{1}$. If both $a(s)$ and $b(s)$ are non-zero, then the solution is the sum of a westward-propagating disturbance of the form (20) and a singular solution with eastward group velocity which is discontinuous at the critical layer. The former does not contribute to the nonlinear dynamics of the critical layer; it is the latter solution that is of interest. Let us therefore consider the case $a(s)=0, b(s) \neq 0$. In that case, the contribution to the solution from the residue at the pole $s=$ $-i l c$ is $e^{-i l c t} \phi_{b}\left(x-x_{c}\right)\left\{\phi_{b}\left(x_{1}-x_{c}\right)\right\}^{-1}$. This is added to the contributions from the branch points to give an approximate solution of (13). In this paper, we only present the solution in the outer region, i.e. away from the critical layer. 
In the outer region where $\left|x-x_{c}\right| t \gg 1$, and for $t \gg 1, \psi$ is found to leading order to be

$$
\begin{array}{r}
\psi(x, y, t) \sim \frac{e^{i l y}}{2}\left\{e^{-i l c t} \phi_{\infty}(x)+e^{-i l x t} h_{1}(x) t^{-2-i \gamma}+h_{2}(x) t^{i \gamma}+\right. \\
\left.+\left(t^{-1}\right)\right\} \\
+ \text { c.c. },(21)
\end{array}
$$

where

$$
\begin{gathered}
\phi_{\infty}(x)=\frac{\phi_{b}\left(x-x_{c}\right)}{\phi_{b}\left(x_{1}-x_{c}\right)}, \\
h_{1}(x) \sim\left(x-x_{c}\right)^{-1}\left(x_{1}-x\right)^{-1-i \gamma}
\end{gathered}
$$

and

$$
h_{2}(x) \sim\left(x_{1}-x_{c}\right)^{-1}\left(x_{1}-x\right)^{1+i \gamma}
$$

The outer solution breaks down as $x \rightarrow x_{c}$. In the critical layer where $\left|x-x_{c}\right| t \sim$ $O(1), t \gg 1$ and $\left|x-x_{c}\right| \ll 1$, an inner solution is derived and matched to the outer solution. The outer and inner solutions of the linear equation can be used as a starting point for deriving an approximate solution of the nonlinear equation (3) following the procedure used by [2]. The detailed derivation of the linear and nonlinear analytic solutions will be given in a subsequent paper.

\section{Numerical solution of the nonlinear equation}

The nonlinear equation (3) is solved numerically in a rectangular domain in the $x y$-plane. The numerical methods used are based on those of [4]. The numerical solution of the linear equation (4) was described in [3] for a case where the forcing took the form of a wave packet localized in the $y$-direction and comprising a continuous spectrum of meridional wavenumbers. In the present study, the forcing comprises a single meridional wavenumber. At the forced boundary $x=x_{1}$, the streamfunction is set to $\psi=\cos l(y-c t)$. As in [3], the basic velocity is set $\bar{v}(x)=\tanh x$. At the other boundary $x=x_{2}$, two types of boundary conditions are employed. In cases where the disturbance is transmitted through the critical layer, a radiation condition, which is described in [3], is used. In cases where there is a negligible amount of transmission, it suffices to set $\psi$ to zero at the outflow boundary. Periodic boundary conditions are assumed at the southern and northern boundaries of the computational domain, which are at $y=-2 \pi$ and $y=2 \pi$, respectively.

The parameters $\beta, \delta, l$ and $c$ are chosen to satisfy the requirements that the disturbance be propagating without decay away from the forced boundary and that any part of the disturbance transmitted beyond the critical layer decays. If $v_{1}$ denotes the local value of $\bar{v}(x)$ near the forced boundary $x=x_{1}$ and $v_{2}$ denotes 


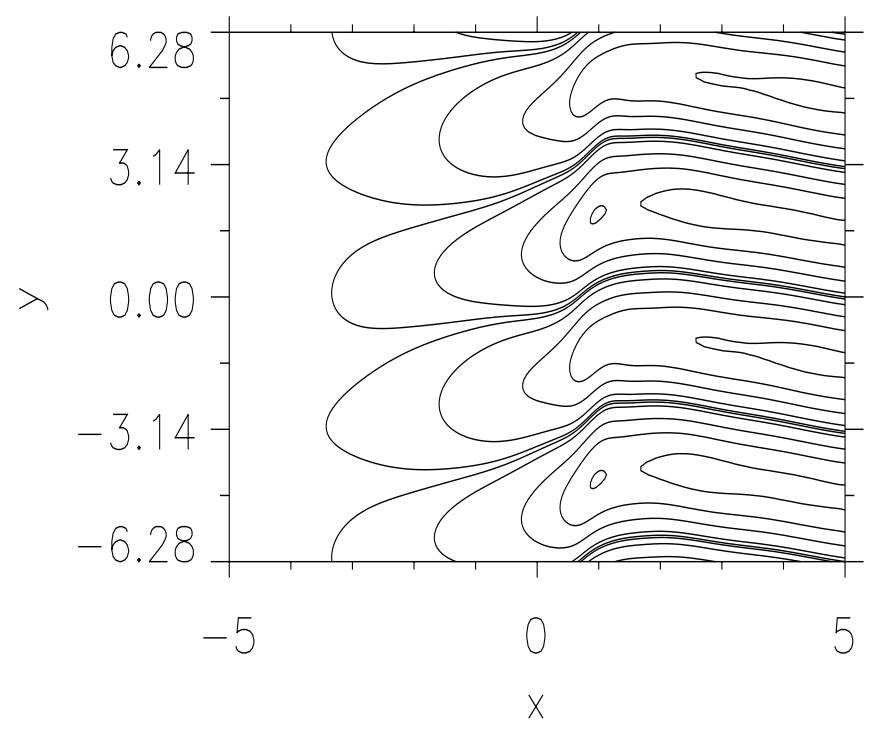

Figure 1: Nonlinear simulation of a westward-propagating Rossby wave forced to the east of its critical layer: Perturbation streamfunction $\psi$ at $t=200$.

the local value at the opposite boundary $x=x_{2}$, then these conditions lead to the inequality

$$
\delta^{1 / 2} l^{2}\left|v_{1}-c\right| \leq \beta<2 \delta^{1 / 2} l^{2}\left|v_{2}-c\right| .
$$

For the hyperbolic tangent profile used here, this means that when the forcing is imposed at the western boundary so that $v_{1}=-1$ and $v_{2}=1$, then $c$ must be negative, and when the forcing is imposed at the western boundary so that $v_{1}=1$ and $v_{2}=-1$, then $c$ must be positive. We therefore set $c=\tanh (-1)$ in the simulations with a eastward-propagating wave and $c=\tanh (1)$ in the simulations with a westward-propagating wave. We also set $\beta=1, \delta=1$ and $l=1$.

According to the linear solution (20), a westward-propagating wave forced to the east of the critical layer passes through. Figure 1 shows the result of a nonlinear simulation for this configuration. The amplitude parameter $\varepsilon$ has been set to 0.02 . Contours of the perturbation streamfunction are shown at time $t=200$. The critical line is located at $x=1$. As in the linear case, the wave passes through the critical layer. With this choice of input parameters, the amplitude of the transmitted wave decays from the critical layer to the outflow boundary. Thus, although the radiation condition is linear, it continues to work up until about $t=350$. Around this time, the wave amplitude becomes large near the outflow boundary and numerical instabilities develop. To continue the simulations beyond this time a nonlinear radiation condition would be needed.

Figure 2 shows the results of a nonlinear simulation in which the wave is forced at the western boundary of the domain. The critical line is located at $x=-1$. At 
(a)

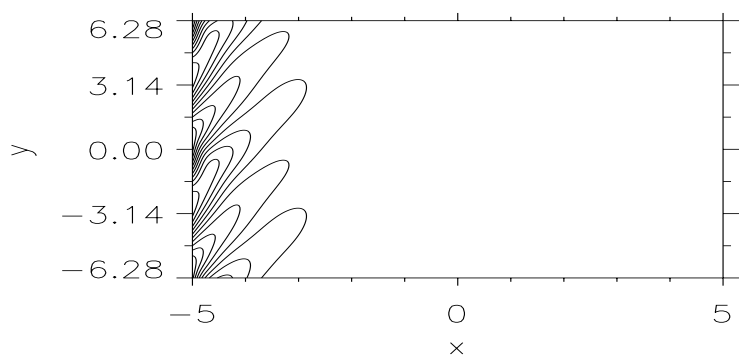

(b)

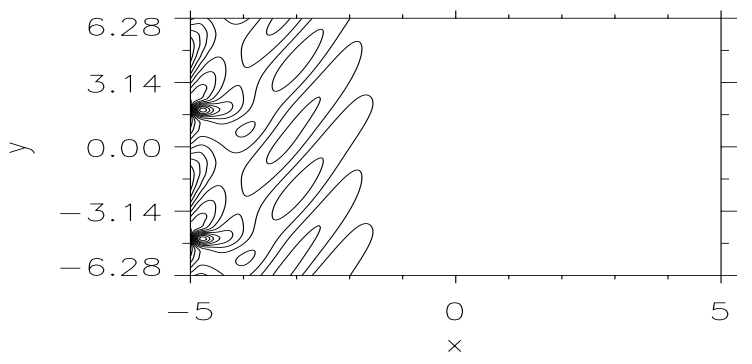

Figure 2: Nonlinear simulation of an eastward-propagating Rossby wave forced to the west of its critical layer: Perturbation streamfunction $\psi$ at (a) $t=20$, (b) $t=200$.

early time (Figure 2(a)) the wave is completely absorbed at the critical layer, as predicted by the linear solution. Continuing the simulation to $t=200$ (Figure 2(b)), we see evidence of a reflected wave near the forced boundary.

We can verify that wave reflection is indeed taking place in Figure 2(b) by evaluating the discontinuity across the critical layer of the meridional average of the zonal momentum flux. This is defined as $F(x, t)=\left(\overline{\psi_{x} \psi_{y}}\right)$, where the overbar denotes an average taken over a meridional wavelength $2 \pi / l$. The difference $[F]$ in this quantity between two points on either side of the critical layer is analogous to the "Reynolds stress jump" in the more familiar case of southward-propagating waves in a zonal shear flow. The evolution of $[F]$ with time is shown in Figure 3. Negative values correspond to time regimes in which the waves are being absorbed, while zero and positive values indicate critical layer reflection. The graph shows that the critical layer alternates between these states.

\section{Conclusions}

This paper discussed the critical-layer dynamics of a Rossby wave propagating longitudinally in a north-south shear flow. An approximate solution of the governing linearized equation was presented. Numerical solutions of the governing nonlinear equation were described. These solutions show that an eastward-propagating wave incident on the critical layer from the west is absorbed by the mean flow at 


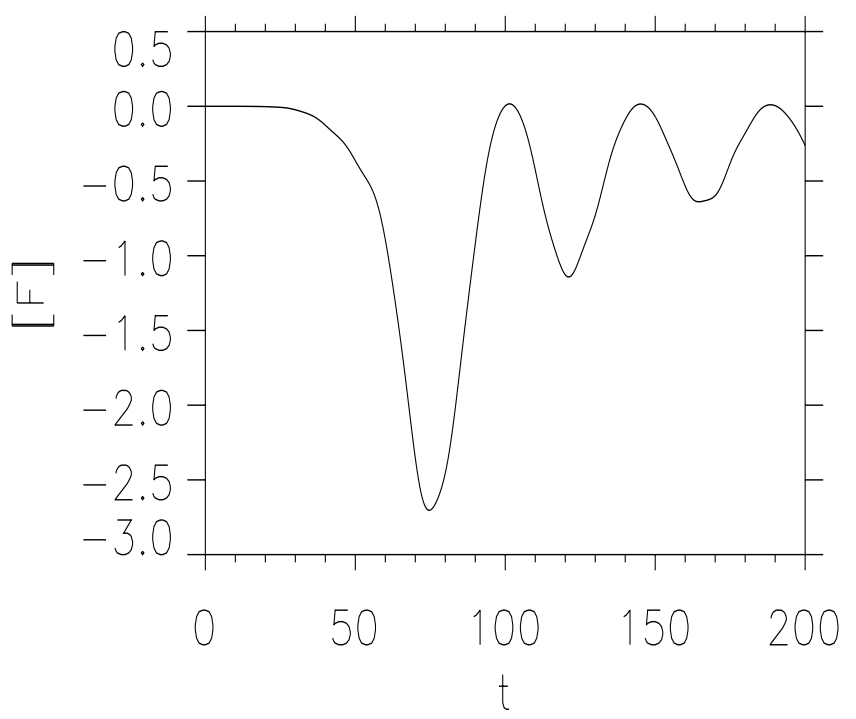

Figure 3: Nonlinear simulation of an eastward-propagating Rossby wave forced to the west of its critical layer: Momentum flux discontinuity $[F]$ plotted as a function of $t$.

early times, but at later time the wave may be reflected from the critical layer. On the other hand, a westward-propagating wave incident on the critical layer from the east passes through the critical layer.

\section{References}

[1] Béland, M., Numerical study of the nonlinear Rossby wave critical level development in a barotropic zonal flow, J. Atmos. Sci., 33 pp. 2066-2078, 1976.

[2] Campbell, L.J., Wave-mean-flow interactions in a forced Rossby wave packet critical layer, Stud. Appl. Math, 112, pp. 39-85, 2004.

[3] Campbell, L.J. \& Maslowe, S.A., Forced Rossby wave packets in barotropic shear flows with critical layers, Dyn. Atmos. Oceans, 28, pp. 9-37, 1998.

[4] Campbell, L.J. \& Maslowe, S.A., A numerical simulation of the nonlinear critical layer evolution of a forced Rossby wave packet in a zonal shear flow, Math. Comp. in Simulation 55, pp. 365-375, 2001.

[5] Dickinson, R.E., Development of a Rossby wave critical level, J. Atmos. Sci., 27, pp. 627-633, 1970.

[6] Fantini, M. \& Tung, K.K., On radiating waves generated from barotropic instability of a western boundary current, J. Phys. Oceanography, 17, pp. 1304-1308, 1979. 
[7] Geisler, J.E. \& Dickinson, R.E., Critical level absorption of barotropic Rossby waves in a north-south flow, J. Geophys. Res., 80, pp. 3805-3811, 1975.

[8] Ierley, G.R., Young, W.R., Viscous instabilities in the western boundary layer, J. Phys. Oceanography, 21, pp. 1323-1332, 1991.

[9] Stewartson, K., The evolution of the critical layer of a Rossby wave, Geophys. Astrophys. Fluid Dyn., 9, pp. 185-200, 1978.

[10] Warn, T. \& Warn, H., On the development of a Rossby wave critical level, J. Atmos. Sci., 33, pp. 2021-2024, 1976.

[11] Warn, T. \& Warn, H., The evolution of a nonlinear critical level, Stud. Appl. Math., 59, pp. 37-71, 1978. 